\title{
CONOCIMIENTO Y ACEPTACIÓN DE ALIMENTOS GENÉTICAMENTE MODIFICADOS EN CONSUMIDORES DE LA IX REGIÓN DE CHILE ${ }^{1}$
}

\author{
KNOWLEDGE AND ACCEPTANCE OF GENETICALLY MODIFIED \\ FOODS AMONG CONSUMERS IN THE IX ${ }^{\text {th }}$ REGION OF CHILE
}

\author{
Berta Schnettler Morales²; Oriana Sepúlveda Bravo²; Danilo Ruiz Fuentes ${ }^{2}$
}

\begin{abstract}
RESUMEN
Considerando el debate generado por los alimentos genéticamente modificados (AGM) en los países desarrollados, se determinó el grado de conocimiento sobre AGM y la importancia de esta variable en la decisión de compra de consumidores de Temuco (Chile), mediante una encuesta personal a 400 personas. Se obtuvo un grado de conocimiento del tema inferior a la media. Mediante análisis conjunto se determinó en leche fluida y salsa de tomate, en general, que la presencia de modificación genética en el alimento fue más importante que la marca y el precio en la decisión de compra. Los consumidores prefirieron alimentos no transgénicos y de marcas conocidas, pero no están dispuestos a pagar un mayor precio por alimentos no transgénicos.
\end{abstract}

Palabras clave: Comportamiento de compra, alimentos transgénicos, análisis conjunto.

\begin{abstract}
In the context of the debate about genetically modified foods (GMF) generated in developed countries, the degree of knowledge about GMF and the importance of this variable in the purchase decision of consumers in Temuco (Chile) was determined by means of a personal survey of 400 individuals. The degree of knowledge of the subject found was below average. Using conjoint analysis, it was found that in liquid milk and tomato sauce, in general, the presence of genetic modification in the food was more important than the brand and the price in the purchase decision. Consumers preferred non-transgenic foods, of known brands, but were not prepared to pay a higher price for non-transgenic foods.
\end{abstract}

Key words: Purchasing behaviour, genetically modified foods, conjoint analysis.

\section{INTRODUCCIÓN}

Un alimento transgénico o genéticamente modificado (AGM) se define como aquel cuyos ingredientes incluyen un organismo genéticamente modificado, el cual a su vez es cualquier vegetal, animal $\mathrm{u}$ organismo cuyo material genético ha sido modificado por el hombre de manera intencional. No existe en el mundo una política común en cuanto e este tipo de alimentos. Chile no tiene una legislación que explícitamente prohíba o condicione la comercialización y consumo de alimentos transgénicos. Tampoco existe un cuerpo jurídico sistematizado, sólo disposiciones sectoriales en materia agrícola, de salud, pesca, medio ambiente, etc.

La actitud del consumidor hacia los AGM está determinada por el riesgo y beneficios percibidos, los que se encuentran determinados en general por el conocimiento que se tiene sobre los AGM (Verdume y Viaene, 2003; Hossain et al., 2003). Beneficios sustanciales, como un menor precio (Lusk et al., 2002; Noussair et al., 2002; Hossain et al., 2003) o un mayor valor nutritivo (Frewer et al., 1999; Einsiedel, 2000; IFIC, 2001), compensarían el riesgo percibido resultando en una actitud positiva hacia los transgénicos. Sin embargo, Einsiedel (2000)

1 Financiado por la Universidad de La Frontera, Proyecto DIUFRO No 120601

2 Universidad de La Frontera, Facultad de Ciencias Agropecuarias y Forestales, Casilla 54-D, Temuco, Chile. E-mail: bschnett@ufro.cl

Fecha de Recepción: 26 Marzo 2007

Fecha de Aceptación: 6 Abril 2007 
determinó que $53 \%$ de los consumidores europeos y $54 \%$ de los canadienses están dispuestos a pagar un mayor precio por alimentos no transgénicos. En forma similar, Larue et al. (2004) determinaron que los consumidores canadienses prefieren los productos convencionales por sobre alimentos similares producidos con manipulación genética. Mientras Grunert et al. (2000) determinaron que los consumidores de los países nórdicos generalmente poseen una actitud negativa hacia los AGM, la que no logra ser compensada con atributos relacionados con una mayor funcionalidad del alimento. Roosen et al. (2003) determinaron que el consumidor europeo no sólo se preocupa del consumo directo de AGM, sino que también del consumo indirecto, por ejemplo, el uso de AGM en producción animal. Verdume y Viaene (2003) señalan que los consumidores están más dispuestos a comprar AGM de marcas conocidas respecto de alimentos transgénicos genéricos, porque las marcas conocidas les brindan confianza. El nivel de confianza es muy relevante para la aceptación de los AGM, puesto que altos niveles de desconfianza aumentan la percepción de riesgo y disminuyen la aceptación del consumidor (Gaskell et al., 1999). Por otra parte, mientras algunas investigaciones dan cuenta de una mayor disposición a adquirir AGM en hombres y personas de mediana edad (Frewer et al., 1999; Einsiedel, 2000; Gamble et al., 2000; Mendenhall y Evenson, 2000; Verdume y Viaene, 2003; Hossain et al., 2003), otras indican que la aceptación de los AGM no posee relación con las características socioeconómicas y demográficas de los consumidores (Lusk et al., 2001; Hossain y Onyango, 2004).

Con base en estos antecedentes, el objetivo de este estudio fue determinar el grado de conocimiento sobre alimentos transgénicos, la importancia de atributos relevantes del producto (precio, marca) y la condición de transgénico de un alimento en la decisión de compra y diferencias entre distintos segmentos de población de la ciudad de Temuco, IX Región de Chile.

\section{MATERIALES Y MÉTODOS}

Se realizó una encuesta personal a una muestra de 400 consumidores de la ciudad de Temuco, IX Región de Chile, cuyo número se obtuvo mediante la fórmula de muestreo aleatorio simple para poblaciones no finitas ( $\mathrm{N}>100.000$; Temuco: 245.347 habitantes al Censo de 2002), considerando 95\% de confianza y $5 \%$ de error de estimación con $\mathrm{p}$ y q de 0,5 (Fernández, 2002). Como instrumento de recogida de información se utilizó un cuestionario con preguntas cerradas. Se incluyeron tres preguntas respecto al grado de conocimiento de los encuestados sobre alimentos importados. La primera pregunta ¿Ud. ha escuchado, visto o leído algo sobre alimentos transgénicos? se realizó para verificar el conocimiento sobre el tema. La segunda pregunta consultó si el encuestado sabía qué significa que un alimento sea transgénico. $\mathrm{La}$ tercera pregunta, consistente en cinco afirmaciones de verdadero o falso, tuvo como objetivo medir cuán correcto era el conocimiento sobre el uso de la biotecnología y los alimentos transgénicos (Los cultivos agrícolas pueden hacerse resistentes a enfermedades o plagas mediante la modificación de su material genético. La manipulación genética no se usa en medicina. Las características de animales no pueden ser transmitidas genéticamente a las plantas. En forma contraria a los alimentos tradicionales, los transgénicos poseen genes. En Chile no se comercializan alimentos transgénicos). Para cada afirmación, los encuestados debieron contestar verdadero o falso y responder cuán seguros estaban de la respuesta usando una escala de siete puntos ( 7 = absolutamente seguro, $1=$ completamente inseguro). Basado en estas respuestas se calculó el grado de conocimiento utilizando la siguiente ecuación (Verdume y Viaene, 2003):

$$
G C=\left\{\left[\sum_{i=1}^{5}\left(A_{i} \times C_{i}\right)\right] / 5\right\} \times 100
$$

donde $\mathrm{GC}=$ grado de conocimiento $(\%) ; \mathrm{A}_{\mathrm{i}} \in\{0,1\}$; $\mathrm{C}_{\mathrm{i}} \in\{0,00 ; 0,17 ; 0,33 ; 0,50 ; 0,67 ; 0,83 ; 1,00\}$. Si la respuesta $(\mathrm{V}$ o $\mathrm{F})$ fue correcta/incorrecta se le asignó puntuación $1 / 0\left(\mathrm{~A}_{\mathrm{i}}\right)$. El factor de certeza 0,00 se asignó si dentro de la escala de 7 puntos se eligió "uno"; 0,17 si se eligió "dos"; 0,33 si se eligió "tres"; 0,50 al elegir "cuatro"; 0,67 al seleccionar "cinco"; 0,83 al seleccionar "seis" y 1,00 si se eligió "siete". El GC se obtuvo dividiendo la suma de los cinco $\left(\mathrm{A}_{\mathrm{i}} \times \mathrm{C}_{\mathrm{i}}\right)$ por cinco y multiplicando este resultado por 100.

Para determinar la importancia de la condición de transgénico de un alimento en la decisión de compra se utilizó análisis conjunto que corresponde a un método descomposicional que permite estimar la importancia relativa de los atributos de un producto y 
estimar valores parciales de utilidad para cada nivel de un atributo (Harrison et al., 2001). Las utilidades estimadas indican cuán influyente es cada nivel de un atributo en la formación de preferencias de los consumidores para una combinación en particular, es decir, representan el grado de preferencia por cada nivel de cada atributo (Hair et al., 1999). Este análisis fue realizado para determinar la importancia de los atributos presencia o ausencia de manipulación genética, marca y precio en la compra de leche fluida y salsa de tomate; la elección de estos alimentos tuvo como propósito evaluar la importancia de la existencia de modificación genética en el consumo indirecto y directo de alimentos transgénicos. Los niveles definidos para la presencia o ausencia de manipulación genética en ambos alimentos fueron: transgénico y no transgénico. Dentro de marca se definieron marcas nacionales y marcas propias; entendiéndose como marca propia o privada aquella creada para, controlada por y/o vendida por los minoristas, mientras un producto de marca vendido por el fabricante a través de minoristas es una marca nacional (Sethuraman y Cole, 1999). Como marcas nacionales se usó Nestlé en leche y Malloa en salsa de tomate. Los niveles de precio fueron establecidos en base al precio promedio del mercado de Temuco al momento de la encuesta (\$400/L leche fluida y $\$ 165 /$ envase de $200 \mathrm{~g}$ de salsa de tomates), sobre el cual se adicionó y restó aproximadamente $10 \%$. De esta forma los niveles en leche fluida fueron $\$ 440 / \mathrm{L}$ y $\$ 360 / \mathrm{L}$, y en salsa de tomate $\$ 180$ /envase de 200 g y $\$ 150 /$ envase de 20 g. A partir de estos atributos y niveles se obtuvieron 8 combinaciones ( $2 \times 2 \times 2)$ identificadas con una letra desde la A hasta la $\mathrm{H}$ en cada alimento. En leche fluida las combinaciones fueron: A) transgénica-Nestlé- $\$ 440 / \mathrm{L}$, B) transgénica-Nestlé-\$360/L, C) transgénicamarca propia- $\$ 440 / \mathrm{L}, \mathrm{D})$ transgénica-marca propia-\$360/L, E) no transgénica-Nestlé-\$440/L, F) no transgénica-Nestlé- $\$ 360 / \mathrm{L}, \mathrm{G}$ ) no transgénicamarca propia- $\$ 440 / \mathrm{L} \mathrm{y,} \mathrm{H)} \mathrm{no} \mathrm{transgénica-marca}$ propia- $\$ 360 / \mathrm{L}$. En salsa de tomate las combinaciones fueron: A) transgénica-Malloa- $\$ 180 / 200 \mathrm{~g}, \mathrm{~B}$ ) transgénica-Malloa-\$150/200 g, C) transgénica-marca propia-\$180/200 g, D) transgénica-marca propia$\$ 150 / 200 \mathrm{~g}, \mathrm{E})$ no transgénica-Malloa-\$180/200 g, F) no transgénica-Malloa- $\$ 150 / 200 \mathrm{~g}, \mathrm{G})$ no transgénica-marca propia- $\$ 180 / 200 \mathrm{~g} \mathrm{y,} \mathrm{H)} \mathrm{no}$ transgénica-marca propia-\$150/200 g. La función de preferencia correspondió al Modelo de Punto Ideal. Para la recogida de datos se usó el procedi- miento de perfil total, para lo cual se elaboraron ocho tarjetas en forma respectiva, con una especificación para cada atributo. A los encuestados se les solicitó que ordenaran las tarjetas desde la más preferida hasta la menos preferida usando una escala de 1 a 8 ( 1 = más preferida; $8=$ menos preferida). Para el atributo precio se estableció una relación lineal, debido a que generalmente a mayor precio la utilidad o preferencia es menor. Los atributos restantes fueron considerados como variables discretas. El análisis se realizó para la muestra completa y submuestras obtenidas según las variables sociodemográficas usadas y el grado de conocimiento (GC) sobre AGM. Para estimar la bondad de ajuste del modelo se utilizó correlación de Pearson (Hair et al., 1999).

Basado en los resultados del AC, se calculó la disposición a pagar marginal (DAP-Mg) por un alimento sin manipulación genética. De acuerdo a Gan y Luzar (1993), la DAP-Mg se estima por medio del cuociente negativo entre el coeficiente (valor parcial de utilidad) de cada nivel de un atributo y el coeficiente del atributo precio (valor $\beta$ ). El procedimiento de cálculo es el siguiente:

$$
D A P-M g=-\left(\frac{\text { Utilidad notransgénico }}{\beta \text { precio }}\right)
$$

Finalmente, la encuesta incluyó preguntas de clasificación de los encuestados: género, edad, zona de residencia, presencia de hijos y su edad, estilo de vida, ocupación y estudios del jefe de hogar y la tenencia de 10 bienes domésticos, estas dos últimas variables para determinar el grupo socioeconómico según Adimark (2004), correspondiente a ABC1 (alto y medio alto), C2 (medio-medio), C3 (medio-bajo), D (bajo) y E (muy bajo). La encuesta se aplicó en dos supermercados de Temuco entre abril y junio de 2006, posterior a la validación del cuestionario mediante un pretest con $10 \%$ de la muestra. Los resultados de las preguntas de conocimiento de alimentos transgénicos fueron analizados mediante Tablas de Contingencia según las características sociodemográficas de los encuestados y el estadístico Chi Cuadrado, que permite inferir si dos o más magnitudes de frecuencias de casos de la población pueden ser consideradas similares (Levin y Rubin, 1996). Se usó el programa SPSS 14.0 (SPSS. Inc., USA) para Windows, que para análisis conjunto presenta el modelo de estimación de parámetros correspondiente a MANOVA. 


\section{RESULTADOS Y DISCUSIÓN}

\section{CONOCIMIENTO SOBRE ALIMENTOS TRANSGÉNICOS}

En el Cuadro 1 se presenta la composición porcentual de la muestra de consumidores encuestados, según las variables de clasificación sociodemográfica utilizadas. En el mismo cuadro se observa que 59\% de los encuestados de la muestra total ha tenido algún tipo de información sobre AGM. Se observaron diferencias significativas respecto a la muestra total según la edad, zona de residencia, presencia de hijos y su edad $(\mathrm{p} \leq 0,05)$ y grupo socioeconómico $(\mathrm{p} \leq 0,001)$ del consumidor, debido al mayor porcentaje de personas menores de 35 años, consumidores sin hijos y pertenecientes al grupo socioeconómico $\mathrm{ABC} 1$ que ha tenido información sobre AGM y la menor proporción de consumidores residentes en zonas rurales, personas con hijos entre 5 y 12 años y pertenecientes a los estratos D y E, en la misma condición. Cabe señalar que ninguno de los encuestados del grupo $\mathrm{E}$ indicó haber recibido información sobre AGM, sin embargo, la representatividad de esta submuestra es baja $(n=9)$. En forma paralela, se obtuvo que sólo $26,3 \%$ de la muestra total indicó conocer lo que significa que un alimento sea transgénico, con diferencias significativas según la zona de residencia, grupo socioeconómico $(\mathrm{p} \leq 0,05)$, edad del consumidor y presencia y edad de los hijos ( $\mathrm{p} \leq 0,001)$. Las diferencias se debieron al mayor porcentaje de personas menores de 35 años, sin hijos y pertenecientes al grupo socioeconómico $\mathrm{ABC} 1$ que indicó conocer el significado, mientras la situación contraria se observó en personas entre 35 y 54 años, residentes en zonas rurales, personas con hijos entre 5 y 12 años y pertenecientes a los estratos $\mathrm{D}$ y $\mathrm{E}$.

La determinación del grado de conocimiento (GC) sobre biotecnología y alimentos transgénicos a través de cinco afirmaciones de verdadero y falso sobre el tema arrojó un nivel inferior a la media correspondiente a un GC de $45,5 \%$ en la muestra total. Los mayores valores de GC correspondieron a personas menores de 35 años, sin hijos, del estrato $\mathrm{ABC} 1$ y en "otra situación laboral", mientras los consumidores entre 35 y 54 años, residentes en zonas rurales, cesantes y pertenecientes a los grupos D y E, obtuvieron los menores GC. En la muestra total $18,8 \%$ de los encuestados presentó un GC igual o menor a $25 \%$; $45,3 \%$ tuvo un GC entre
26 y $50 \% ; 24,3 \%$ entre 51 y $75 \%$ y $11,8 \%$ obtuvo un GC entre 76 y $100 \%$. Se obtuvieron diferencias estadísticamente significativas según la zona de residencia $(\mathrm{p} \leq 0,05)$, edad del consumidor, edad de los hijos y grupo socioeconómico ( $\mathrm{p} \leq 0,001)$. Estas se debieron a la mayor proporción de personas menores de 35 años que tuvo un GC superior a $75 \%$ y mayor porcentaje de personas entre $35 \mathrm{y}$ 54 años con GC entre 26 y $50 \%$. De acuerdo a la zona de residencia debido al mayor porcentaje de personas que viven en zonas rurales que presentó un GC igual o menor a $25 \%$, mientras según la presencia y edad de los hijos destacó la alta cifra de personas sin hijos que obtuvo un GC superior a $75 \%$ y la mayor fracción de personas con hijos entre 5 y 12 años con GC entre 0 y 25\%. Según el grupo socioeconómico, los estratos de mayores ingresos ( $\mathrm{ABC} 1 \mathrm{y} \mathrm{C} 2)$ presentaron mayor porcentaje de consumidores con GC entre 51 y $75 \%$, mientras en los grupos más bajos (D y E) la cifra de personas con GC igual o menor a $25 \%$ fue elevada. La frase que registró mayor proporción de respuestas correctas en la muestra total fue "Los cultivos agrícolas pueden hacerse resistentes a enfermedades o plagas mediante la modificación de su material genético" (verdadero) con $82 \%$, seguida por las frases "En Chile no se comercializan alimentos transgénicos" (falsa) y "La manipulación genética no se usa en medicina" (falsa) con cifras en torno a 75\%. La frase "En forma contraria a los alimentos tradicionales, los alimentos transgénicos poseen genes" (falsa) registró $60 \%$ de respuestas correctas, mientras "Las características de animales no pueden ser transmitidas genéticamente a las plantas" (falsa) fue contestada correctamente por $43 \%$.

\section{IMPORTANCIA DE LA CONDICIÓN DE TRANSGÉNICO DEL ALIMENTO EN LA DECI- SIÓN DE COMPRA}

Como se observa en el Cuadro 2, en la muestra total se obtuvo que el factor de mayor importancia en la compra de ambos alimentos fue la presencia o ausencia de manipulación genética $(44,7 \%$ en leche y $44,3 \%$ en salsa de tomate), seguido por la marca $(29,8 \%$ en leche y $30,0 \%$ en salsa de tomate) y el precio $(25,5 \%$ en leche y $25,7 \%$ en salsa de tomate), lo que se contrapone a lo determinado por Baker y Burnham (2002) en EE.UU. donde el precio fue más importante que la presencia de modificación genética en el alimento y la marca 
Cuadro 1

Composición de la muestra y conocimiento sobre AGM (\%). Temuco, enero de 2007

\begin{tabular}{|c|c|c|c|c|c|c|c|c|}
\hline \multirow{2}{*}{ Muestra } & \multirow{2}{*}{ Muestra (\%) } & \multirow{2}{*}{ Información AGM } & \multirow{2}{*}{ Significado AGM } & \multirow{2}{*}{ GC medio } & \multicolumn{4}{|c|}{ Rango GC } \\
\hline & & & & & $0-25$ & $26-50$ & $51-75$ & $76-100$ \\
\hline Total & 100 & $\mathbf{5 9 , 0}$ & 26,3 & 45,5 & 18,8 & 45,3 & 24,3 & 11,8 \\
\hline \multicolumn{2}{|l|}{ Género } & $P=0,297$ & $P=0,761$ & \multicolumn{5}{|c|}{$P=0,936$} \\
\hline Masculino & 48,3 & 61,7 & 26,9 & 44,4 & 19,7 & 45,1 & 24,4 & 10,9 \\
\hline Femenino & 51,8 & 56,5 & 25,6 & 46,5 & 17,9 & 45,4 & 24,2 & 12,6 \\
\hline \multicolumn{2}{|l|}{ Edad } & $P=\mathbf{0 , 0 1 3}$ & $\mathbf{P}=\mathbf{0 , 0 0 0}$ & \multicolumn{5}{|c|}{$\mathbf{P}=\mathbf{0 , 0 0 0}$} \\
\hline$<35$ años & 33,3 & 68,4 & 37,6 & 52,7 & 13,5 & 39,8 & 24,1 & 22,6 \\
\hline 35-54 años & 45,3 & 51,9 & 17,7 & 39,9 & 22,7 & 50,8 & 22,1 & 4,4 \\
\hline 55 años y más & 21,5 & 59,3 & 26,7 & 46,0 & 18,6 & 41,9 & 29,1 & 10,5 \\
\hline \multicolumn{2}{|l|}{ Residencia } & $P=\mathbf{0 , 0 3 0}$ & $\mathbf{P}=\mathbf{0 , 0 3 8}$ & \multicolumn{5}{|c|}{$P=0,005$} \\
\hline Urbana & 80,8 & 61,6 & 28,5 & 46,9 & 15,8 & 47,1 & 23,8 & 13,3 \\
\hline Rural & 19,3 & 48,1 & 16,9 & 39,5 & 31,2 & 37,7 & 26,0 & 5,2 \\
\hline \multicolumn{2}{|l|}{ Edad hijos } & $P=0,004$ & $\mathbf{P}=\mathbf{0 , 0 0 0}$ & \multicolumn{5}{|c|}{$P=0,000$} \\
\hline Sin hijos & 28,3 & 70,8 & 42,5 & 53,3 & 13,3 & 37,2 & 24,8 & 24,8 \\
\hline Hijos $<5$ años & 9,0 & 55,6 & 25,0 & 46,6 & 13,9 & 52,8 & 22,2 & 11,1 \\
\hline Hijos 5-12 años & 10,0 & 37,5 & 10,0 & 34,7 & 35,0 & 40,0 & 25,0 & 0 \\
\hline Hijos $13-17$ años & 30,3 & 59,5 & 19,0 & 42,5 & 20,7 & 52,1 & 19,8 & 7,4 \\
\hline Hijos $\geq 18$ años & 22,5 & 54,4 & 23,0 & 43,9 & 17,8 & 45,6 & 30,0 & 6,7 \\
\hline \multicolumn{2}{|l|}{ Ocupación } & $P=0,059$ & $P=0,217$ & \multicolumn{5}{|c|}{$P=\mathbf{0 , 5 7 8}$} \\
\hline Cta, propia & 22,0 & 44,3 & 18,2 & 40,4 & 28,4 & 40,9 & 19,3 & 11,4 \\
\hline Empresario & 10,5 & 64,3 & 31,0 & 43,2 & 21,4 & 42,9 & 28,6 & 7,1 \\
\hline Emp, particular & 29,8 & 64,7 & 27,7 & 47,4 & 15,1 & 47,1 & 25,2 & 12,6 \\
\hline Emp, Público & 19,3 & 62,3 & 24,7 & 47,4 & 19,5 & 40,3 & 26,0 & 14,3 \\
\hline Jubilado & 12,5 & 56,0 & 38,0 & 48,3 & 10,0 & 54,0 & 24,0 & 12,0 \\
\hline Cesante & 2,3 & 77,7 & 11,1 & 36,9 & 22,2 & 66,7 & 11,1 & 0 \\
\hline Otra situación & 3,8 & 66,7 & 26,7 & 51,3 & 6,7 & 46,7 & 33,3 & 13,3 \\
\hline \multicolumn{2}{|l|}{ GSE } & $P=0,000$ & $P=0,002$ & \multicolumn{5}{|c|}{$P=0,000$} \\
\hline $\mathrm{ABC} 1$ & 34,8 & 68,3 & 34,5 & 50,4 & 12,9 & 43,2 & 29,5 & 14,4 \\
\hline $\mathrm{C} 2$ & 33,0 & 62,9 & 29,5 & 49,0 & 12,1 & 44,7 & 30,3 & 12,9 \\
\hline C3 & 17,3 & 56,5 & 17,4 & 39,3 & 21,7 & 55,1 & 17,4 & 5,8 \\
\hline $\mathrm{D}$ & 12,8 & 37,3 & 11,8 & 33,5 & 43,1 & 39,2 & 5,9 & 11,8 \\
\hline $\mathrm{E}$ & 2,3 & 0 & 0 & 32,6 & 44,4 & 44,4 & 11,1 & 0 \\
\hline \multicolumn{2}{|l|}{ Estilo de vida } & $P=0,567$ & $P=0,616$ & \multicolumn{5}{|c|}{$P=0,766$} \\
\hline Conservador & 36,0 & 54,9 & 25,7 & 43,3 & 19,4 & 50,0 & 22,9 & 7,6 \\
\hline Liberal & 36,3 & 60,7 & 25,5 & 46,9 & 20,0 & 41,4 & 24,8 & 13,8 \\
\hline Vegetariano & 10,8 & 60,5 & 23,3 & 43,5 & 20,9 & 46,5 & 23,3 & 9,3 \\
\hline Deportista & 9,3 & 64,9 & 32,4 & 49,4 & 13,5 & 37,8 & 32,4 & 16,2 \\
\hline Innovador & 7,8 & 57,1 & 25,0 & 45,6 & 14,3 & 50,0 & 17,9 & 17,9 \\
\hline
\end{tabular}


Cuadro 2

Importancia (\%) de los atributos presencia de modificación genética, marca y precio en el proceso de decisión de compra de leche y salsa de tomates. Temuco, enero de 2007

\begin{tabular}{|c|c|c|c|c|c|c|c|c|}
\hline \multirow{2}{*}{ Muestra } & \multicolumn{4}{|c|}{ Leche } & \multicolumn{4}{|c|}{ Salsa de tomate } \\
\hline & MG & Marca & Precio & R Pearson & MG & Marca & Precio & R Pearson \\
\hline Total & 44,7 & 29,8 & 25,5 & 0,999 & 44,3 & 30,0 & 25,7 & 0,998 \\
\hline Masculino & 44,3 & 29,9 & 25,8 & 1,000 & 43,4 & 29,8 & 26,8 & 0,998 \\
\hline Femenino & 45,1 & 29,7 & 25,2 & 0,997 & 45,2 & 30,1 & 24,7 & 0,997 \\
\hline$<35$ años & 46,4 & 27,9 & 25,7 & 0,999 & 47,4 & 28,9 & 23,7 & 0,999 \\
\hline 35-54 años & 43,5 & 30,8 & 25,7 & 0,999 & 43,3 & 30,6 & 26,1 & 0,996 \\
\hline 55 años y más & 44,4 & 30,6 & 25,0 & 0,997 & 41,7 & 30,3 & 28,0 & 0,997 \\
\hline Urbana & 46,2 & 29,4 & 25,4 & 0,998 & 45,3 & 29,8 & 24,9 & 0,998 \\
\hline Rural & 38,6 & 31,2 & 30,2 & 0,997 & 40,1 & 30,8 & 29,1 & 0,998 \\
\hline Sin hijos & 45,2 & 29,6 & 25,2 & 1,000 & 45,8 & 29,6 & 24,7 & 1,000 \\
\hline Hijos $<5$ años & 50,2 & 26,4 & 23,4 & 0,996 & 51,4 & 28,9 & 19,7 & 0,995 \\
\hline Hijos 5-12 años & 41,1 & 29,9 & 29,0 & 0,986 & 42,1 & 29,5 & 28,4 & 0,986 \\
\hline Hijos 13-17 años & 43,4 & 30,8 & 25,8 & 0,998 & 43,7 & 30,9 & 25,4 & 0,990 \\
\hline Hijos $\geq 18$ años & 45,2 & 30,8 & 24,8 & 0,996 & 41,5 & 29,9 & 28,6 & 0,998 \\
\hline Cta. Propia & 44,1 & 29,9 & 26,0 & 1,000 & 43,2 & 30,2 & 26,6 & 0,999 \\
\hline Empresario & 48,9 & 29,3 & 21,8 & 0,959 & 46,7 & 27,9 & 25,4 & 0,975 \\
\hline Emp. Particular & 46,2 & 29,1 & 24,7 & 0,997 & 45,4 & 29,9 & 24,7 & 0,992 \\
\hline Emp. Público & 43,8 & 29,6 & 26,6 & 0,998 & 46,4 & 27,0 & 26,6 & 0,998 \\
\hline Jubilado & 45,7 & 28,9 & 25,4 & 0,997 & 43,2 & 30,8 & 26,0 & 0,996 \\
\hline Cesante & 34,9 & 35,7 & 29,4 & 0,999 & 38,1 & 35,7 & 26,2 & 0,999 \\
\hline Otra situación & 31,7 & 35,9 & 32,4 & 0,966 & 32,7 & 43,7 & 23,6 & 0,996 \\
\hline $\mathrm{ABC} 1$ & 46,9 & 28,0 & 25,1 & 0,995 & 46,0 & 28,8 & 25,2 & 0,993 \\
\hline $\mathrm{C} 2$ & 43,7 & 31,7 & 24,6 & 0,999 & 44,5 & 31,3 & 24,2 & 0,999 \\
\hline $\mathrm{C} 3$ & 43,6 & 29,3 & 27,1 & 0,998 & 40,9 & 29,3 & 29,8 & 0,996 \\
\hline $\mathrm{D}$ & 42,9 & 29,9 & 27,2 & 1,000 & 44,0 & 29,3 & 26,7 & 0,999 \\
\hline $\mathrm{E}$ & 43,2 & 31,2 & 25,6 & 0,995 & 43,7 & 38,1 & 18,2 & 0,991 \\
\hline Conservador & 48,1 & 28,2 & 23,7 & 1,000 & 48,2 & 27,8 & 24,0 & 0,999 \\
\hline Liberal & 42,1 & 30,7 & 27,2 & 0,999 & 41,5 & 30,9 & 27,6 & 0,995 \\
\hline Vegetariano & 49,5 & 27,2 & 23,3 & 0,989 & 46,5 & 28,2 & 25,3 & 0,982 \\
\hline Deportista & 38,1 & 34,8 & 27,1 & 0,981 & 38,9 & 36,2 & 24,9 & 1,000 \\
\hline Innovador & 41,8 & 30,0 & 28,2 & 0,999 & 41,6 & 31,7 & 26,7 & 0,999 \\
\hline Sabe significado & 46,7 & 28,5 & 24,8 & 0,998 & 46,2 & 27,8 & 26,0 & 0,998 \\
\hline No sabe significado & 43,9 & 30,3 & 25,8 & 0,999 & 43,7 & 30,7 & 25,6 & 0,998 \\
\hline GC $0-25 \%$ & 43,9 & 30,0 & 26,2 & 0,993 & 43,0 & 29,5 & 27,5 & 0,995 \\
\hline GC $26-50 \%$ & 44,0 & 29,8 & 26,2 & 0,999 & 43,8 & 30,4 & 25,8 & 0,999 \\
\hline GC $51-75 \%$ & 44,4 & 30,5 & 25,1 & 0,999 & 44,0 & 30,0 & 26,0 & 0,997 \\
\hline GC $76-100 \%$ & 49,1 & 28,0 & 22,9 & 0,999 & 49,1 & 29,0 & 21,9 & 1,000 \\
\hline
\end{tabular}

MG: Presencia o ausencia de manipulación genética en el alimento. 
como atributos evaluados en el proceso de decisión de compra de cereales para el desayuno. Si bien el orden de importancia se mantuvo en la mayoría de las submuestras analizadas, destacaron en leche los cesantes y personas "en otra situación laboral”, para quienes la marca tuvo levemente mayor importancia que los restantes atributos, lo que según Baker y Burnham (2002) indica que ninguno de los atributos domina las preferencias del consumidor. En salsa de tomates, claramente la marca tuvo mayor importancia que la presencia de modificación genética y el precio en las personas "en otra situación laboral". La existencia de manipulación genética en leche presentó mayor importancia en las submuestras correspondientes a personas con hijos menores de 5 años, empresarios, personas con estilo de vida vegetariano y consumidores con un grado de conocimiento (GC) sobre alimentos transgénicos mayor a $75 \%$, con ponderaciones en torno a 50\%. Los menores valores de importancia correspondieron a personas residentes en zonas rurales, cesantes, consumidores en otra situación laboral y de estilo de vida deportista, con pesos inferiores a 39\%. En salsa de tomate, sólo las personas con hijos menores a 5 años y las personas con GC entre 76 y $100 \%$ presentaron valores de importancia en torno a $50 \%$, mientras nuevamente las cifras más bajas se registraron en cesantes, personas en "otra situación laboral" y deportistas. Cabe destacar que en ambos alimentos la importancia de la manipulación genética fue mayor en los consumidores que indicaron conocer el significado de "alimento transgénico". Los coeficientes de correlación de Pearson fueron cercanos a 1 y estadísticamente significativos $(\mathrm{p} \leq 0,001)$ en todas las muestras analizadas, lo que indica una buena bondad de ajuste del modelo conjunto.

\section{UTILIDAD DE LOS ATRIBUTOS Y DISPOSICIÓN A PAGAR POR UN ALIMENTO SIN MANIPULACIÓN GENÉTICA}

Como se observa en el Cuadro 3, en la muestra total el consumidor experimentó utilidad positiva o preferencia hacia la leche producida sin manipulación genética y negativa respecto a la presencia de manipulación genética, ambas de la misma magnitud pero de signo contrario, resultado que fue similar en la mayoría de las submuestras a excepción de las personas con hijos entre 13 y 17 años y los cesantes, que presentaron el comporta- miento contrario. Similares fueron los resultados en salsa de tomate, con utilidad positiva frente al producto no transgénico y utilidad negativa o rechazo hacia el producto transgénico, presentando el comportamiento opuesto sólo la submuestra de cesantes (Cuadro 4). Esto concuerda con la preferencia de alimentos no transgénicos determinada por Larue et al. (2004) en Canadá y con la actitud negativa de los consumidores nórdicos hacia los alimentos transgénicos reportada por Grunert et al. (2000). La mayor preferencia por leche y salsa de tomate sin manipulación genética y rechazo por estos productos transgénicos correspondió a las personas menores de 35 años, sin hijos, jubilados, pertenecientes al estrato socioeconómico D y consumidores con GC entre 76 y $100 \%$. Si se considera que la actitud del consumidor se relaciona con el conocimiento sobre los AGM (Verdume y Viaene, 2003), es posible indicar que un mayor grado de conocimiento sobre el tema genera un mayor rechazo por los alimentos transgénicos o una mayor preferencia por los alimentos sin manipulación genética. El grupo de consumidores con GC mayor a $75 \%$ presentó diferencias significativas respecto a la muestra total $(\mathrm{p} \leq 0,001)$ en la proporción de personas que había tenido información sobre AGM (93,6\%), que previamente conocía el significado de "alimento transgénico" (80,9\%), por estar formado por un alto porcentaje de personas menores a 35 años $(63,8 \%)$, consumidores sin hijos $(59,6 \%)$ y del estrato ABC1 $(42,6 \%)$.

La utilidad o pérdida de utilidad en forma respectiva en ambos alimentos fue mayor en mujeres y en personas que previamente conocían el significado de AGM respecto de los hombres y de las que desconocían el significado. En la mayoría de las submuestras se obtuvo una pérdida de utilidad mayor en el caso de la leche producida con manipulación genética, excepto en personas menores de 35 años, con hijos entre 0 y 17 años, empleados particulares, jubilados, cesantes, en otra situación laboral, pertenecientes al grupo C3, deportistas, personas que conocían el significado de "alimento transgénico" y con GC superior a $75 \%$, para quienes comparativamente la pérdida de utilidad fue mayor en el caso de salsa de tomate transgénica. Esto permite señalar que los consumidores se preocupan tanto del consumo directo de AGM como del consumo indirecto, asociado al uso de AGM en producción animal, en forma concordante con los resultados de Roosen et al. (2003). 
Cuadro 3

Utilidad de los niveles de los atributos presencia de modificación genética, marca y precio en el proceso de decisión de compra de leche y disposición marginal a pagar (DAP-Mg) por leche sin modificación genética (\%). Temuco, enero de 2007

\begin{tabular}{|c|c|c|c|c|c|c|c|c|}
\hline \multirow{2}{*}{ Muestra } & \multicolumn{2}{|c|}{ Manipulación genética } & \multicolumn{2}{|c|}{ Marca } & \multicolumn{2}{|c|}{ Precio } & \multirow{2}{*}{$\beta$ precio } & \multirow{2}{*}{ DAP Mg } \\
\hline & CMG & SMG & Nestlé & Propia & $\$ 360$ & $\$ 440$ & & \\
\hline Total & $-0,569$ & 0,659 & $\mathbf{0 , 7 7 7}$ & $-0,777$ & -0964 & -1928 & $-0,964$ & 100,15 \\
\hline Masculino & $-0,464$ & 0,464 & 0,798 & $-0,798$ & $-0,956$ & $-1,912$ & $-0,956$ & 100,12 \\
\hline Femenino & $-0,667$ & 0,667 & 0,757 & $-0,757$ & $-0,971$ & $-1,942$ & $-0,971$ & 100,17 \\
\hline$<35$ años & $-1,017$ & 1,017 & 0,836 & $-0,836$ & $-1,169$ & $-2,338$ & $-1,169$ & 100,22 \\
\hline 35-54 años & $-0,329$ & 0,329 & 0,753 & $-0,753$ & $-0,895$ & $-1,790$ & $-0,895$ & 100,09 \\
\hline 55 años y más & $-0,381$ & 0,381 & 0,735 & $-0,735$ & $-0,791$ & $-1,581$ & $-0,791$ & 100,12 \\
\hline Urbana & $-0,557$ & 0,557 & 0,754 & $-0,754$ & $-0,910$ & $-1,820$ & $-0,910$ & 100,15 \\
\hline Rural & $-0,617$ & 0,617 & 0,873 & $-0,873$ & $-1,188$ & $-2,377$ & $-1,188$ & 100,13 \\
\hline Sin hijos & $-1,374$ & 1,374 & 0,934 & $-0,934$ & $-1,434$ & $-2,867$ & $-1,434$ & 100,24 \\
\hline Hijos $<5$ años & $-0,375$ & 0,375 & 0,715 & $-0,715$ & $-0,931$ & $-1,861$ & $-0,931$ & 100,10 \\
\hline Hijos 5-12 años & $-0,431$ & 0,431 & 0,669 & $-0,669$ & $-1,113$ & $-2,225$ & $-1,113$ & 100,10 \\
\hline Hijos 13-17 años & 0,103 & $-0,103$ & 0,729 & $-0,729$ & $-0,558$ & $-1,116$ & $-0,558$ & 99,95 \\
\hline Hijos $\geq 18$ años & $-0,600$ & 0,600 & 0,717 & $-0,717$ & $-0,867$ & $-1,733$ & $-0,867$ & 100,17 \\
\hline Cta. propia & $-0,676$ & 0,676 & 0,864 & $-0,864$ & $-1,080$ & $-2,159$ & $-1,080$ & 100,16 \\
\hline Empresario & $-0,113$ & 0,113 & 0,542 & $-0,542$ & $-0,190$ & $-0,381$ & $-0,190$ & 100,15 \\
\hline Emp. particular & $-0,620$ & 0,620 & 0,786 & $-0,786$ & $-0,929$ & $-1,857$ & $-0,929$ & 100,17 \\
\hline Emp. público & $-0,503$ & 0,503 & 0,776 & $-0,776$ & $-1,156$ & $-2,312$ & $-1,156$ & 100,11 \\
\hline Jubilado & $-1,025$ & 1,025 & 0,740 & $-0,740$ & $-1,450$ & $-2,900$ & $-1,450$ & 100,18 \\
\hline Cesante & 0,444 & $-0,444$ & 1,139 & $-1,139$ & 0,389 & 0,778 & $-0,389$ & 99.97 \\
\hline Otra situación & $-0,233$ & 0,233 & 0,767 & $-0,767$ & $-0,933$ & $-1,867$ & $-0,933$ & 100,06 \\
\hline $\mathrm{ABC} 1$ & $-0,362$ & 0,362 & 0,721 & $-0,721$ & $-0,701$ & $-1,403$ & $-0,701$ & 100,13 \\
\hline $\mathrm{C} 2$ & $-0,462$ & 0,462 & 0,752 & $-0,752$ & $-0,973$ & $-1,947$ & $-0,973$ & 100,12 \\
\hline $\mathrm{C} 3$ & $-0,710$ & 0,710 & 0,746 & $-0,746$ & $-1,326$ & $-2,652$ & $-1,326$ & 100,13 \\
\hline D & $-1,191$ & 1,191 & 0,990 & $-0,990$ & $-1,088$ & $-2,176$ & $-1,088$ & 100,27 \\
\hline $\mathrm{E}$ & $-0,722$ & 0,722 & 1,028 & $-1,028$ & $-1,389$ & $-2,778$ & $-1,389$ & 100,13 \\
\hline Conservador & $-0,920$ & 0,920 & 0,707 & $-0,707$ & $-1,080$ & $-2,160$ & $-1,080$ & 100,21 \\
\hline Liberal & $-0,321$ & 0,321 & 0,833 & $-0,833$ & $-1,134$ & $-2,269$ & $-1,134$ & 100,07 \\
\hline Vegetariano & $-0,343$ & 0,343 & 0,581 & $-0,581$ & $-0,407$ & $-0,814$ & $-0,407$ & 100,21 \\
\hline Deportista & $-0,466$ & 0,466 & 0,959 & $-0,959$ & $-0,216$ & $-0,432$ & $-0,216$ & 100,54 \\
\hline Innovador & $-0,411$ & 0,411 & 0,866 & $-0,866$ & $-1,357$ & $-2,714$ & $-1,357$ & 100,08 \\
\hline Sabe significado & $-0,807$ & 0,807 & 0,781 & $-0,781$ & $-1,300$ & $-2,600$ & $-1,300$ & 100,16 \\
\hline No sabe sign. & $-0,484$ & 0,484 & 0,775 & $-0,775$ & $-0,844$ & $-1,688$ & $-0,844$ & 100,14 \\
\hline GC $0-25 \%$ & $-0,670$ & 0,670 & 0,740 & $-0,740$ & $-1,253$ & $-2,507$ & $-1,253$ & 100,13 \\
\hline GC $26-50 \%$ & $-0,390$ & 0,390 & 0,749 & $-0,749$ & $-0,790$ & $-1,580$ & $-0,790$ & 100,12 \\
\hline GC $51-75 \%$ & $-0,631$ & 0,631 & 0,858 & $-0,858$ & $-0,928$ & $-1,856$ & $-0,928$ & 100,17 \\
\hline GC 76-100\% & $-0,968$ & 0,968 & 0,777 & $-0,777$ & $-1,245$ & $-2,489$ & $-1,245$ & 100,19 \\
\hline
\end{tabular}

CMG: con manipulación genética. SMG: Sin manipulación genética.

Cifras de utilidad en los distintos niveles de un atributo con signo negativo indican pérdida de utilidad para el consumidor. En el atributo precio, cifras más negativas indican mayor pérdida de utilidad. 


\section{Cuadro 4}

Utilidad de los niveles de los atributos presencia de modificación genética, marca y precio en la compra de salsa de tomate y disposición marginal a pagar (DAP-Mg) por salsa de tomate sin modificación genética (\%). Temuco, enero de 2007

\begin{tabular}{|c|c|c|c|c|c|c|c|c|}
\hline \multirow{2}{*}{ Muestra } & \multicolumn{2}{|c|}{ Manipulación genética } & \multirow{2}{*}{$\begin{array}{l}\text { Marca } \\
\text { Malloa }\end{array}$} & \multirow{2}{*}{$\begin{array}{c}\text { Propia } \\
-0,746\end{array}$} & \multicolumn{2}{|c|}{ Precio } & \multirow{2}{*}{$\beta$ precio } & \multirow{2}{*}{ DAP Mg } \\
\hline & CMG & SMG & & & $\$ 150$ & $\$ 180$ & & \\
\hline Total & $-0,634$ & 0,634 & 0,746 & $-0,741$ & $-0,971$ & $-1,943$ & $-0,971$ & 100,40 \\
\hline Masculino & $-0,508$ & 0,508 & 0,741 & $-0,751$ & $-1,060$ & $-2,119$ & $-1,060$ & 100,29 \\
\hline Femenino & $-0,751$ & 0,751 & 0,751 & $-0,797$ & $-0,889$ & $-1,778$ & $-0,889$ & 100,51 \\
\hline$<35$ años & $-1,000$ & 1,000 & 0,797 & $-0,750$ & $-1,117$ & $-2,233$ & $-1,117$ & 100,54 \\
\hline 35-54 años & $-0,434$ & 0,434 & 0,750 & $-0,660$ & $-0,945$ & $-1,890$ & $-0,945$ & 100,28 \\
\hline 55 años y más & $-0,488$ & 0,488 & 0,660 & $-0,660$ & $-0,802$ & $-1,605$ & $-0,802$ & 100,37 \\
\hline Urbana & $-0,606$ & 0,606 & 0,733 & $-0,733$ & $-0,906$ & $-1,811$ & $-0,906$ & 100,41 \\
\hline Rural & $-0,750$ & 0,750 & 0,802 & $-0,802$ & $-1,247$ & $-2,494$ & $-1,247$ & 100,36 \\
\hline Sin hijos & $-1,418$ & 1,418 & 0,894 & $-0,894$ & $-1,473$ & $-2,947$ & $-1,473$ & 100,58 \\
\hline Hijos $<5$ años & $-0,340$ & 0,340 & 0,729 & $-0,729$ & $-0,750$ & $-1,500$ & $-0,750$ & 100,27 \\
\hline Hijos 5-12 años & $-0,413$ & 0,413 & 0,600 & $-0,600$ & $-1,100$ & $-2,200$ & $-1,100$ & 100,23 \\
\hline Hijos 13-17 años & $-0,054$ & 0,054 & 0,709 & $-0,709$ & $-0,607$ & $-1,215$ & $-0,607$ & 100,05 \\
\hline Hijos $\geq 18$ años & $-0,644$ & 0,644 & 0,683 & $-0,683$ & $-0,861$ & $-1,722$ & $-0,861$ & 100,45 \\
\hline Cta. propia & $-0,750$ & 0,750 & 0,776 & $-0,766$ & $-1,000$ & $-2,000$ & $-1,000$ & 100,45 \\
\hline Empresario & $-0,375$ & 0,375 & 0,601 & $-0,601$ & $-0,738$ & $-1,476$ & $-0,738$ & 100,31 \\
\hline Emp. particular & $-0,603$ & 0,603 & 0,796 & $-0,796$ & $-0,929$ & $-1,857$ & $-0,929$ & 100,39 \\
\hline Emp. público & $-0,656$ & 0,656 & 0,747 & $-0,747$ & $-0,948$ & $-1,896$ & $-0,948$ & 100,42 \\
\hline Jubilado & $-1,005$ & 1,005 & 0,720 & $-0,720$ & $-1,340$ & $-2,680$ & $-1,340$ & 100,45 \\
\hline Cesante & 0,222 & $-0,222$ & 0,694 & $-0,694$ & $-0,722$ & $-1,444$ & $-0,722$ & 99,81 \\
\hline Otra situación & $-0,083$ & 0,083 & 0,700 & $-0,700$ & $-0,833$ & $-1,667$ & $-0,833$ & 100,06 \\
\hline $\mathrm{ABC} 1$ & $-0,547$ & 0,547 & 0,664 & $-0,664$ & $-0,727$ & $-1,453$ & $-0,727$ & 100,46 \\
\hline $\mathrm{C} 2$ & $-0,491$ & 0,491 & 0,739 & $-0,739$ & $-1,061$ & $-2,121$ & $-1,061$ & 100,28 \\
\hline $\mathrm{C} 3$ & $-0,649$ & 0,649 & 0,783 & $-0,783$ & $-1,196$ & $-2,391$ & $-1,196$ & 100,33 \\
\hline $\mathrm{D}$ & $-1,196$ & 1,196 & 0,863 & $-0,863$ & $-1,118$ & $-2,235$ & $-1,118$ & 100,65 \\
\hline $\mathrm{E}$ & $-0,788$ & 0,788 & 1,194 & $-1,194$ & $-0,889$ & $-1,778$ & $-0,889$ & 100,54 \\
\hline Conservador & $-0,934$ & 0,934 & 0,743 & $-0,743$ & $-1,017$ & $-2,035$ & $-1,017$ & 100,56 \\
\hline Liberal & $-0,412$ & 0,412 & 0,762 & $-0,762$ & $-1,148$ & $-2,297$ & $-1,148$ & 100,22 \\
\hline Vegetariano & $-0,512$ & 0,512 & 0,599 & $-0,599$ & $-0,558$ & $-1,116$ & $-0,558$ & 100,56 \\
\hline Deportista & $-0,358$ & 0,358 & 0,858 & $-0,858$ & $-0,419$ & $-0,838$ & $-0,419$ & 100,52 \\
\hline Innovador & $-0,643$ & 0,643 & 0,750 & $-0,750$ & $-1,214$ & $-2,429$ & $-1,214$ & 100,32 \\
\hline Sabe significado & $-0,774$ & 0,774 & 0,757 & $-0,757$ & $-1,343$ & $-2,686$ & $-1,343$ & 100,00 \\
\hline No sabe signif. & $-0,584$ & 0,584 & 0,742 & $-0,742$ & $-0,839$ & $-1,678$ & $-0,839$ & 100,42 \\
\hline GC $0-25 \%$ & $-0,847$ & 0,847 & 0,760 & $-0,760$ & $-1,313$ & $-2,627$ & $-1,313$ & 100,39 \\
\hline GC $26-50 \%$ & $-0,420$ & 0,420 & 0,735 & $-0,735$ & $-0,693$ & $-1,387$ & $-0,693$ & 100,37 \\
\hline GC $51-75 \%$ & $-0,716$ & 0,716 & 0,771 & $-0,771$ & $-1,103$ & $-2,206$ & $-1,103$ & 100,39 \\
\hline GC 76-100\% & $-0,947$ & 0,947 & 0,718 & $-0,718$ & $-1,223$ & $-2,447$ & $-1,223$ & 100,47 \\
\hline
\end{tabular}

CMG: con manipulación genética. SMG: Sin manipulación genética.

Cifras de utilidad en los distintos niveles de un atributo con signo negativo indican pérdida de utilidad para el consumidor. En el atributo precio, cifras más negativas indican mayor pérdida de utilidad. 
Tanto en la muestra total como en las submuestras estudiadas en ambos alimentos se obtuvo utilidad positiva frente a las marcas nacionales (Nestlé en leche y Malloa en salsa de tomate) y pérdida de utilidad o rechazo por las marcas propias, lo que se relaciona con la influencia del riesgo percibido en la compra de un alimento con marca propia (Rajeev e Indrajit, 2000), y concuerda con Verdume y Viaene (2003) respecto a que los consumidores están más dispuestos a comprar alimentos transgénicos de marcas conocidas. Ambos niveles de precio reportaron pérdidas de utilidad en la muestra total y submuestras analizadas en leche y salsa de tomate, las que se incrementaron (cifras de utilidad más negativas) al acceder a comprar los productos a un mayor precio ( $\$ 440 / \mathrm{L}$ en leche y $\$ 180 /$ envase de $200 \mathrm{~g}$ en salsa de tomate), lo que indica que no existe una asociación entre precio y calidad. En forma congruente, las combinaciones con mayor proporción de primera preferencia fueron leche no transgénica-Nestlé-\$360/L $(47,3 \%)$ y salsa de tomate no transgénica-Malloa-\$150/envase de 200 g $(48,3 \%)$, mientras las menos preferidas fueron leche transgénica-marca propia- $\$ 440 / \mathrm{L}(48,8 \%)$ y salsa de tomate transgénica-marca propia- $\$ 180 /$ envase de $200 \mathrm{~g}(47,3 \%)$. Las combinaciones leche transgénica-Nestlé-\$360/L y salsa de tomate transgénica-Malloa-\$150/ envase de 200 g sólo concentraron $15 \%$ de la primera preferencia, por lo cual es posible indicar que un menor precio no logró compensar la pérdida de utilidad producida por la presencia de manipulación genética en el alimento, a diferencia de lo reportado por Lusk et al. (2002), Noussair et al. (2002) y Hossain et al. (2003).

\section{LITERATURA CITADA}

ADIMARK. 2004. Mapa socioeconómico de Chile. Disponible en http://www.adimark.cl/medios/estudios/informe_mapa_socioeconomico_de_chile.pdf. Conectado el 20 de octubre de 2005.

BAKER, G. AND T. BURNHAM. 2002. The market for genetically modified foods: consumer characteristics and policy implications. International Food and Agribusiness Management Review 4: 351-360.

EINSIEDEL, E. 2000. Biotechnology and the Canadian public: 1997 and 2000. Report $f$ the Office of Consumer Affaire (Industry Canada) and the Canadian Food Inspection Agency, Calgary, Alberta: Faculty of Communication and Culture, University of Calgary.

FERNÁNDEZ, A. 2002. Investigación y técnicas de mercado. Primera edición. Editorial Esic. Madrid, España. 273 pp.
En los Cuadros 3 y 4 se presenta la disposición marginal a pagar (DAP-Mag) por leche y salsa de tomate sin manipulación genética, expresada en porcentaje respecto al promedio de los dos niveles de precio utilizados $(\$ 400 / \mathrm{L}$ leche y $\$ 165 /$ envase de $200 \mathrm{~g}$ de salsa de tomate). Si bien en la muestra total $\mathrm{y}$ en la mayoría de las submuestras analizadas, excepto en personas con hijos entre 13 y 17 años para leche y en cesantes para ambos productos, la DAP-Mag por productos sin manipulación genética superó $100 \%$, las diferencias son despreciables. Si se consideran los valores de DAP-Mag del grupo con estilo de vida deportista en leche y de los consumidores del estrato $D$ en salsa de tomate, que fueron los que presentaron mayores DAP-Mg $(100,54 \%$ en leche y $100,65 \%$ en salsa de tomate, respectivamente), traducido a precios implica \$402/L leche y \$166/envase de $200 \mathrm{~g}$ de salsa de tomate sin manipulación genética. Este resultado se contrapone a lo determinado por Einsiedel (2000), respecto a la disposición de consumidores europeos y canadienses a pagar un mayor precio por alimentos no transgénicos.

Por tanto, los consumidores de Temuco, independientemente del producto, prefieren alimentos no transgénicos con respaldo del nombre de marca de un fabricante y privilegian pagar un menor precio. Sin embargo, la pérdida de utilidad que experimenta el consumidor frente a un alimento transgénico no logra ser compensada por un menor precio. El mayor rechazo hacia los AGM correspondió a personas menores de 35 años, sin hijos, jubilados, del grupo C3, de estilo de vida conservador y con mayor conocimiento sobre AGM. Este último aspecto se relaciona con la edad, zona de residencia, presencia y edad de los hijos y grupo socioeconómico del consumidor.

FREWER, L., D. HEDDERLEY, C. HOWARD AND R. SHEPHERD. 1999. Reactions to information about genetic engineering: impact of source credibility, perceived risk immediacy and persuasive content. Public Understanding of Science 8 (1): 35-50.

GAMBLE, J., S. MUGGLESTON, D. HEDDERLY, T. PARMINTER AND G. VAUGHAM. 2000. Genetic engineering: The public's point of view. HortResearch NZ Client Report No. 000/249. New Zealand: HortResearch NZ, NZ.

GAN, C. AND E. LUZAR. 1993. A Conjoint Analysis in Waterfowl Hunting in Louisiana. Journal of Agricultural and Applied Economics 25 (2): 36-45.

GASKELL, G., BRAUER, M., DURANT, J. AND ALLUM, N. 1999. Worlds apart? The reception of genetically modified foods in Europe and the US. Science, 295: 348-387. 
GRUNERT, K., L. LÄHTEENMÄKI, N. NIELSEN, J. POULSEN, O. UELAND AND A. ÅSTRÖM. 2000. Consumer perception of food products involving genetic modification: results from a qualitative study in four Nordic countries. Working papers No. 72, MAPP-Center for Market Surveillance and Strategy for the Food Sector, Århus, Denmark.

HAIR, J., R. ANDERSON, R. TATHAM Y W. BLACK. 1999. Análisis Multivariante. Otero. Quinta edición. Prentice Hall Internacional. Inc. Madrid, España. 832 pp.

HARRISON, R., J. GILLESPIE AND D. FIELDS. 2001. Theoretical and empirical considerations of eliciting preferences and model estimation in conjoint analysis. Selected Paper, American Agricultural Economics Association Annual Meeting, Chicago IL, USA, August 5-8.

HOSSAIN, F., ONYANGO, B., SCHILLING, B., HALLMAN, W. AND ADELAJA, A. 2003. Product attributes consumer benefits and public approval of genetically modified foods. International Journal of Consumer Studies 27 (5): 353-365.

HOSSAIN, F. AND B. ONYANGO. 2004. Products attribute and consumer acceptance of nutritionally enhanced genetically modified foods. International Journal of Consumer Studies, 28 (3): 255-267.

INTERNATIONAL FOOD INFORMATION COUNCIL (IFIC). 2001. Most Americans can Articulate Expected Benefits of Food Biotechnology. Disponible en http://ific. org/foodinsight/2001/so/biotechbenfi501.cfm. Conectado el 20 de agosto de 2009.

LARUE, B., G. WEST, C. GENDRON AND R. LAMBERT. 2004. Consumer response to functional foods produced by conventional, organic, or genetic manipulation. Agribusiness 20 (2): 155-166.
LEVIN, R. Y D. RUBIN. 1996. Estadística para Administradores. Prentice Hall, Hispanoamericana S.A. Sexta edición. Juárez. México. 1018 pp.

LUSK, J., M. DANIEL, D. MARK AND C. LUSK. 2001. Alternative calibration and auction institutions for predicting consumer willingness to pay for nongenetically modified corn chips. Journal of Agricultural Resource Economics 26 (1): 40-57.

LUSK, J., M. MOORE, L. HOUSE AND B. MORROW. 2002. Influence of brand and type of modification on consumer acceptance of genetically engineered corn chips: a preliminary analysis. International Food and Agribusiness Management Review 4: 320-331.

MENDENHALL, C. AND R. EVENSON. 2002. Willingness to pay a premium for non-genetically modified foods. In: V. Santaniello, R. Evenson and D. Zilberman (Eds.), Market development for genetically modified foods (pp. 55-61). Walingfgord: Cabi Publishing.

NOUSSAIR, C., S. ROBIN AND B. RUFFIEUX. 2002. Do consumers not care about biotech foods or do they just not read the labels? Economics Letters 75 (1): 47-53.

RAJEEV, B. AND S. INDRAJIT. 2000. Consumer-Level Factors Moderating the Success of Private Label Brands. Journal of Retailing 76 (2): 175-191.

ROOSEN, J., J. LUSK AND J. FOX. 2003. Consumer demand for and attitudes toward alternative beef labeling strategies in France, Germany and the UK. Agribusiness 19(1): 77-90.

SETHURAMAN, R. AND C. COLE. 1999. Factors influencing the price premiums that consumers pay for national brands over store brands. Journal of Products and Brand Management 8 (4): 340-351.

VERDUME, A. AND J. VIAENE. 2003. Consumer beliefs and attitude towards genetically modified food: basis for segmentation and implications for communication. Agribusiness 219 (1): 91-113. 